\title{
Replik auf den „Letter to Editor: Series of articles by T. Bertsch and G. Erbacher culminating in Lipoedema - myths and facts, Part 5: European Best Practice of Lipoedema - Summary of the European Lipoedema Forum Consensus"
}

Erneut ein Leserbrief aus den USA und erneut einer, der von einer US-amerikanischen Patientenselbsthilfegruppe getragen wird, diesmal DER ANDEREN in unserer letzten Replik [1] bereits erwähnten Selbsthilfegruppe: „Lipedema Simplified“ [2] bzw. dem damit aufs Engste verwobene „The Lipedema Project“ [3]. Es lohnt sich, einen Blick auf diese sehr ansprechend gestaltete Website zu werfen, um eine Ahnung davon zu haben, welches merkantile, aber auch professionelle Know-how hinter dieser - so die Autoren des Leserbriefs - „Non-Profit“- Organisation steckt. Die Mitgliederzahl dieser Organisation wird mit 21500 beziffert, nachprüfbar war dies für uns nicht, eine entsprechende Referenz wurde nicht genannt.

Eine der 5 Leserbriefverfasserinnen, Catherine Seo, Gründerin und Director von „Lipedema Simplified“, ist auch Gründerin und Co-Director von „The Lipedema Project“ - gemeinsam mit dem Liposuktions-Arzt Mark Smith [3]. Die anderen Mitverfasserinnen sind auch Akteurinnen dieses „Lipedema Projects“, die sich zumindest im Leserbrief alle „President“ oder „Director“ nennen. Auf der Website von „The Lipedema Project“ fungiert lediglich Leslyn Keith, eine Physiotherapeutin und Keto-Coach, als President. Die erstgenannte Verfasserin, Brenda Gold, wird dort als „Emotional Freedom Coach“, Raeann Sparks als „Keto Coach \& Mobility Consultant“ aufgeführt [4]. IngMarie Bohmelin, die sich im Leserbrief ebenfalls „President“ nennt, ist die Leiterin einer - lokalen - schwedischen SH-Gruppe und nicht - wie suggeriert - der nationalen schwedischen Patientenvertretung [5]. IngMarie Bohmelin steht mit ihrer Haltung ohnehin in scharfem Kontrast zur offiziellen nationalen Swedish Lymphedema Association (Svensk Förening för Lymfologi, SFL), die den Erstautor dieser Replik im November 2019 nach Stockholm eingeladen hatte, um über die Mythen und Fakten des Lipödems zu referieren. Der Vorstand der SFL, dem u. a. auch die weltweit renommierten Lymphologen Karin
Johansson sowie Hakan Brorson angehören, hat darüber hinaus auch alle 5 Teile der Artikelserie „Lipödem - Mythen und Fakten" [6-10] inklusive des in Teil 5 enthaltenen European Consensus auf der offiziellen Homepage der SFL verlinkt [11].

Neben den beschriebenen Gemeinsamkeiten - über die wir uns freuen - kritisieren die Leserbriefautoren im Wesentlichen 4 Punkte der Artikelserie bzw. des European Consensus, die sich teilweise mit der Kritik des Leserbriefes von Karen Herbst and friends decken. Wiederholungen in dieser Replik mit der Replik auf den Herbst-Leserbrief [1] lassen sich daher nicht vermeiden. Generell spiegelt auch dieser Leserbrief die US-amerikanische Sichtweise des Lipödems wider, die man auf diese 4 Statements herunterbrechen kann:

\section{Adipositas und psychische Störungen der Patientin mit Lipödem werden (wenn überhaupt) eher als Komorbidi- täten betrachtet, nicht als hochrele- vante Lipödem-aggravierende Begleit- erkrankungen.}

2. Als dicker empfundene Beine (im Leserbrief „Dysmorphia“ genannt) werden - ganz unabhängig von einer Beschwerdesymptomatik - als Lipödem diagnostiziert. Eine Differenzierung in Lipohypertrophie (die beschwerdefreie überproportionale Fettgewebsvermehrung der Beine) und Lipödem wird von der US-amerikanischen Selbsthilfeorganisation abgelehnt.

3. Das Lipödem inkludiert eine lymphatische Dysfunktion; daher müssen entstauende Maßnahmen wie manuelle Lymphdrainagen zur Therapie eingesetzt werden.

4. Bariatrische Chirurgie ist keine empfehlenswerte Therapieoption - auch dann nicht, wenn die Patientin mit Lipödem schwer adipös ist und einen BMI $>40 \mathrm{~kg} / \mathrm{m}^{2}$ aufweist.

\section{Zu 1.}

Die US-amerikanische Patientinnenvertretung schreibt: „We agree with setting aside unsubstantiated claims and assertions from the past but we caution against adopting new hypotheses that have not themselves been scientifically validated, and treating them as established or proven. We prefer to acknowledge comorbidities with lipedema addressing them in the present without ascribing causal relations."

Die beiden im European Consensus on Lipedema genannten wesentlichen Komorbiditäten des Lipödem-Syndroms sind Adipositas sowie Erkrankungen aus dem psychischen Spektrum, wie z. B. Depressionen oder Essstörungen. Zur Adipositas schreibt der Consensus im „Diagnostic Approach“: „Overweight/obesity is an aggravating factor of Lipoedema ... Conclusion: Obesity/weight gain must me focused on “ [10]. Mit diesem Statement wurde keine Kausalität hergestellt, allerdings auf die - von Lipödem-Akteurinnen gerne ignorierte - Adipositas als bedeutsamen und aggravierenden Faktor hingewiesen. Die internationale Datenlage ist hier sehr konsistent. Weit mehr als $80 \%$ der Patientinnen mit der Diagnose Lipödem sind adipös, ca. $50 \%$ der Patientinnen sind sogar morbid adipös, haben also einen $\mathrm{BMI}>40 \mathrm{~kg} / \mathrm{m}^{2}$ [12-17]. Hier die Adipositas als entscheidenden Einflussfaktor auf das Lipödem zu missachten ist vergleichbar mit dem Vorgehen, das eigene Haus brennen zu lassen und stattdessen die Garage zu löschen

Zur psychischen Situation der LipödemPatientin schreibt der Consensus:

„Psychological issues are an additional aspect of Lipoedema. Impact of psychological distress is underestimated. Psychological vulnerability contributes to the amount of pain perception ... Conclusion: Psychological assessment is a must" [10].

Auch hier wurde keine Kausalität postuliert - allerdings auf den profunden Zusammenhang zwischen psychischer Belastung (oft auch manifester psychischer Störung) und der Diagnose Lipödem hingewiesen. In einer Untersuchung der Autoren dieser Replik im Europäischen Zentrum für Lymphologie an 150 Patientinnen mit der gesicherten Diagnose Lipödem-Syndrom präsentierten $80 \%$ der 
Frauen psychische Störungsbilder (meist Depression, Essstörung oder posttraumatische Belastungsstörung) oder eine schwere psychische Beeinträchtigung, wie z. B. Burn-out oder chronische Stressbelastung. Diese Störungsbilder bzw. schweren psychischen Beeinträchtigungen bestanden jedoch bereits vor der Entwicklung Lipödem-assoziierter Schmerzen im Weichteilgewebe [18]. Damit kann - formallogisch - die Erkrankung Lipödem-Syndrom nicht Ursache dieser psychischen Belastung sein, was häufig behauptet wird.

Darüber hinaus ist auch der Einfluss psychischer Belastungen und Krankheiten auf die Schmerzentstehung sowie auf die Schmerzwahrnehmung breit untersucht und sehr konsistent [19-25]. In unserer Untersuchung an Patientinnen mit Lipödem waren Depression und Essstörungen im Vergleich zur Prävalenz dieser psychischen Störungsbilder in der Allgemeinbevölkerung [18] vor allem im 12-Monats-Zeitraum vor der Entstehung der Lipödem-assoziierten Schmerzsymptomatik stark erhöht. Auch hier gilt wieder das Bild des brennenden Hauses: Psychische Erkrankungen und Belastungen müssen bei der Betrachtung des Lipödems berücksichtigt und ins Behandlungskonzept integriert werden.

Manche Patientinnen mit der Diagnose Lipödem mögen noch weitere Komorbiditäten aufweisen (wie z. B. Bluthochdruck, Diabetes mellitus oder ein Lymphödem). Diese haben allerdings keinen vergleichbar relevanten Einfluss auf die Erkrankung Lipödem-Syndrom wie Adipositas und psychische Störungen.

\section{Zu 2.}

Den zweiten Kritikpunkt beginnt die USamerikanische Selbsthilfegruppe mit dem Satz: "Second, we are concerned that in a field where there is so much uncertainty, the Forum members were forced to admit that "Serious scientific data on the patient's perspective do not exist. "This is even more worrying in that „crucial questions" the Forum members articulated were: „What do our patients with lipoedema really SUFFER from?" and "What is the therapeutic goal from the patient's point of view?““

Offensichtlich wurde hier von den Leserbriefautoren übersehen, dass unsere „crucial questions“ rein rhetorischer Natur waren, erfolgte doch die Beantwortung

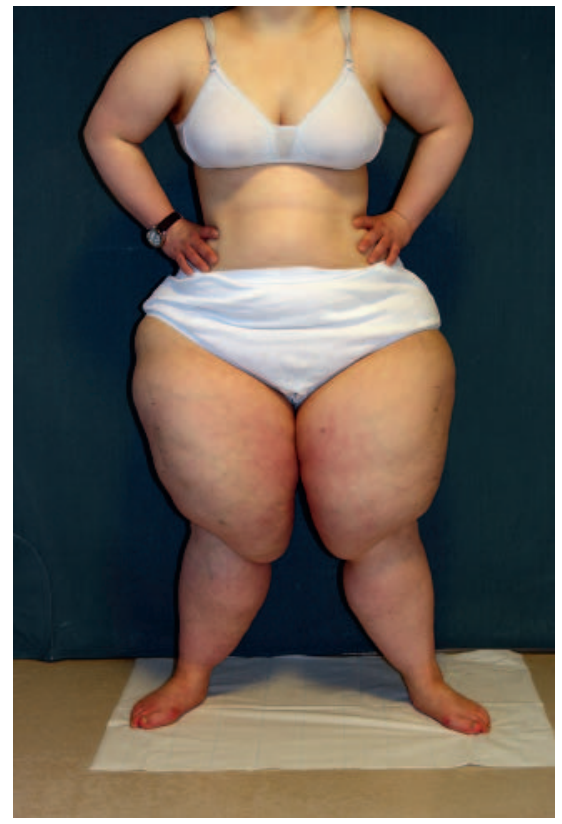

- Abb. 1 Patientin mit Lipödem-Syndrom und ausgeprägter disproportionaler Fettgewebsvermehrung der Beine. Quelle: Dr. Tobias Bertsch, Földiklinik Hinterzarten.

dieser Fragen durch das Expertengremium unmittelbar:

„Patients with lipoedema suffer to varying degrees from:

- pain/other symptoms in the soft tissues of the legs or arms

- greater psychological vulnerability, which may in turn potentiate the pain

- a lack of self-acceptance, mainly because of today's ideal of beauty

- overweight or obesity with numerous attempts at dieting

- a lack of physical exercise and fitness, especially in obese patients“ [10].

Von dieser - auch durchaus in Umfragen an zahlreichen Frauen mit Lipödem bestätigten - Leidenskonstellation ausgehend entwickelte die europäische Expertengruppe dann das dargestellte Therapiekonzept.

Ausführlich widmet sich der Leserbrief dem Begriff „Dysmorphia“, ein Terminus, der in diesem Abschnitt 10-mal aufgeführt wird und damit zeigt, welche Bedeutung dieses Symptom für die US-amerikanische Selbsthilfegruppe hat. Aus deren Sicht stellt die „Dysmorphia“ das einzige und alleinstehende Kriterium zur Diagnosestellung des Lipödems dar. „Dysmorphia is not a cosmetic issue, but a divergence from the

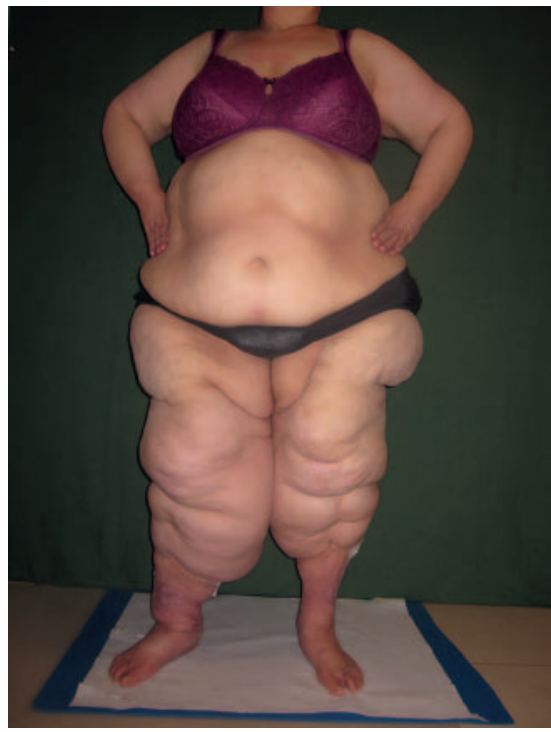

- Abb. 2 Patientin mit morbider Adipositas III $^{\circ}$, Adipositas-assoziierten Beinlymphödemen sowie einem LipödemSyndrom. Quelle: Dr. Tobias Bertsch, Földiklinik Hinterzarten.

anatomically normal that has real, verifiable musculoskeletal consequences in everyday life and in rehabilitation."

Wir bestreiten nicht, dass es Patientinnen gibt, für die diese Beschreibung zutrifft, Patientinnen mit ausgeprägter disproportionaler Fettgewebsvermehrung der Beine. Abb. 1 zeigt eine Patientin, bei der der Terminus „Dysmorphia“ durchaus Anwendung finden kann. Allerdings sehen wir im Europäischen Zentrum für Lymphologie diese Patientinnen nur sehr selten - sicherlich weniger als 5-mal, bei ca. 3500 Patientinnen, die uns jährlich ambulant und stationär mit der Diagnose Lipödem zugewiesen werden. Deutlich häufiger sehen wir Frauen mit der Zuweisungsdiagnose Lipödem wie in $>$ Abb. 2 , für die der Begriff „Dysmorphia“ auch zutreffen könnte, die allerdings 2 weitere lymphologisch relevante und - vorrangig - behandlungsbedürftige Krankheiten aufweisen: eine morbide Adipositas (mit einem BMI von $58 \mathrm{~kg} / \mathrm{m}^{2}$ ) und Adipositasassoziierte Beinlymphödeme.

Die mit Abstand größte Patientinnengruppe unter den uns von hausärztlichen und phlebologischen Kollegen zugewiesenen Frauen mit der Diagnose Lipödem zeigen die $>$ Abb. 3, 4; Patientinnen, bei denen aus unserer Sicht eher die Erkrankung Adipositas als eine (auch additiv) beste- 


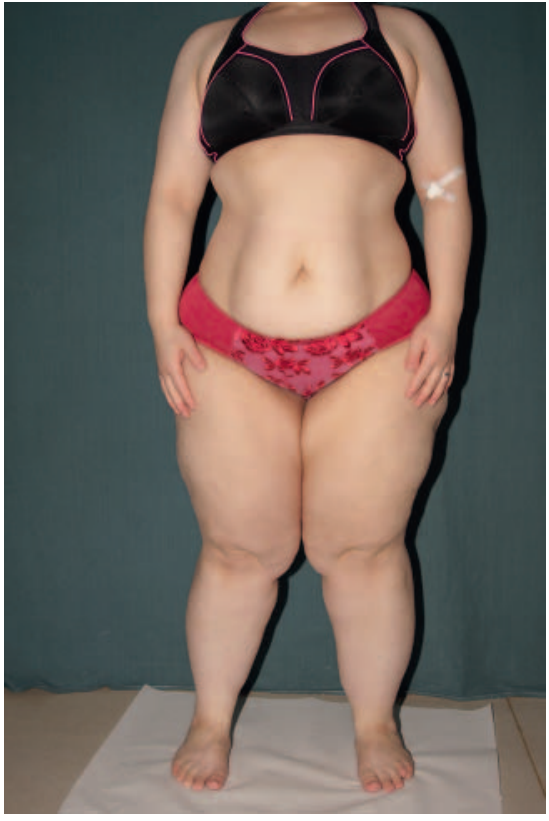

- Abb. 3 Patientin mit Adipositas und Lipödem-Syndrom. Quelle: Dr. Tobias Bertsch, Földiklinik Hinterzarten.

hende Disproportion der Beine ins Auge springt.

Dass das von den Leserbriefschreiberinnen geforderte Konzept der „Dysmorphia“ aber auch durchaus kritisch zu hinterfragen ist, zeigen die - täglich - uns mit der Diagnose Lipödem zugeführten Frauen in - Abb. 5, 6, die einen hohen Leidensdruck aufgrund der subjektiv wahrgenommenen Disproportionalität der Beine aufweisen (oder um im Jargon der Leserbriefschreiber zu bleiben: an „Dysmorphia“ leiden) und eine Befürwortung zur Liposuktion erbitten.

Nebenbei bemerkt: Beide Patientinnen in den Abb. 5, 6 äußerten weder im Rahmen der Anamnese noch bei der klinischen Untersuchung eine Schmerzsymptomatik, sodass wir weder die für die Diagnose Lipödem notwendige Disproportionalität noch das ebenso erforderliche Kriterium „Beschwerden“ klinisch nachvollziehen konnten. Auch diese im Europäischen Consensus definierten Diagnosekriterien werden von den US-amerikanischen Akteurinnen nicht akzeptiert: „we cannot agree with the division of associated dysmorphia into two diagnostic categories: lipedema and lipohypertrophy. “

Noch einmal zurück zum DysmorphiaKonzept der US-Amerikanerinnen: Die Autorinnen des Leserbriefes bleiben die Ant-

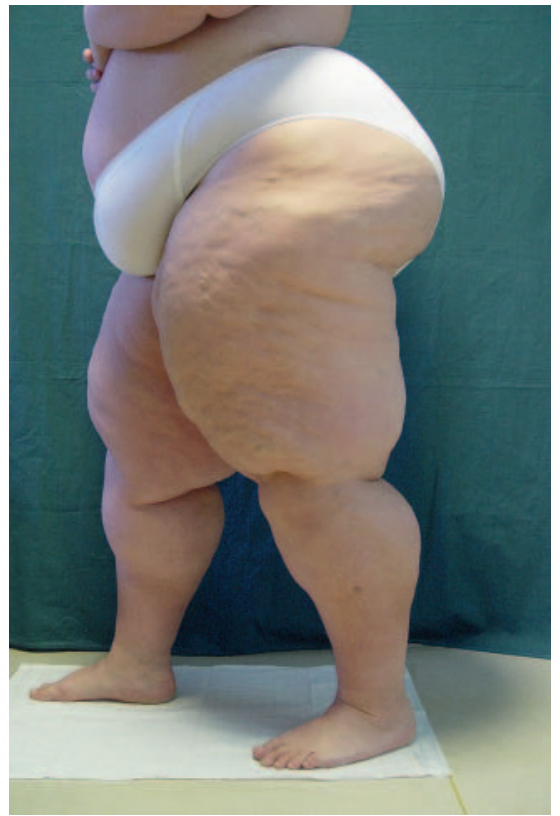

- Abb. 4 Patientin mit morbider Adipositas III ${ }^{\circ}$ und Lipödem-Syndrom. Quelle: Dr. Tobias Bertsch, Földiklinik Hinterzarten.

wort schuldig, welche Kriterien für das Symptom „Dysmorphia“ herangezogen werden sollen, wer letztlich darüber entscheidet, ab wann ein Bein wirklich „dysmorph“ ist. Die Medien durch das von ihnen propagierte Schönheitsideal? Die Patientin selbst? Der absaugende Arzt? Bei Letzterem vermischen sich sicher merkantile Interessen mit medizinischen - was der Medizin selten guttut. Soll die Patientin entscheiden? Nur äußerst wenige Frauen, die der Erstautor dieser Replik in seiner lymphologischen Ambulanz sieht, zeigen - aus subjektiver Sicht des Untersuchers eine dysmorphe Beinkonfiguration. Allerdings empfinden nahezu alle der überwiesenen Frauen die Konfiguration ihrer Beine als disproportional bzw. „dysmorph“. Im Übrigen teilen auch die häufig mitanwesenden Partner der Frauen (die der untersuchende Erstautor regelmäßig mitbefragt) nicht deren kritischen Blick auf die Beine ihrer Partnerinnen.

Während des lymphologischen Weltkongresses (des ILF) 2018 in Rotterdam begann der Erstautor dieser Replik seinen Vortrag zum Thema „Mythen und Fakten des Lipödems“ mit der Frage ins Publikum: „Who of you are happy with your legs?“ Im Publikum saßen etwas 500 Zuhörer, in der Mehrheit Zuhörerinnen - wohlgemerkt keine Patientinnen, sondern Therapeutin-

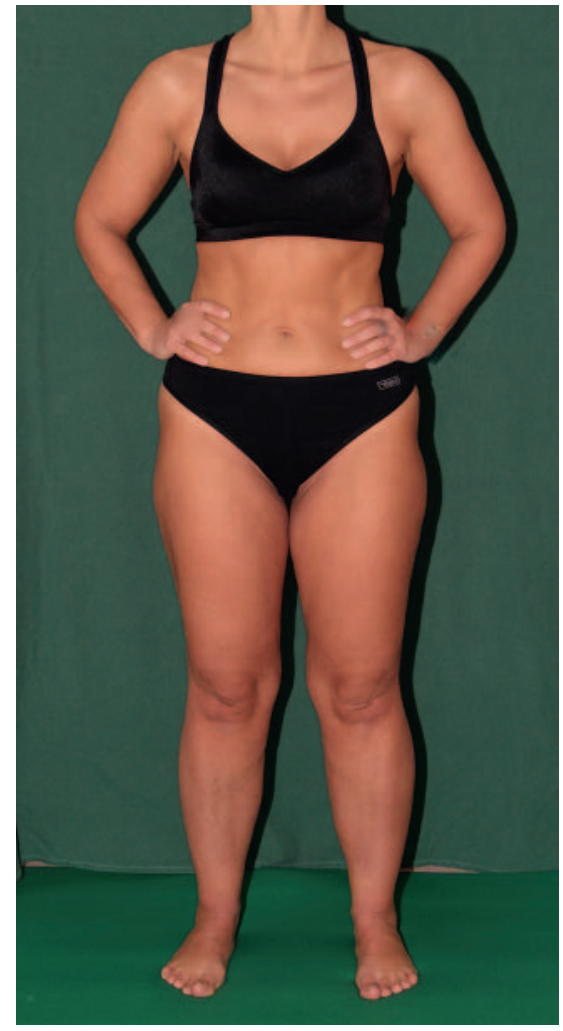

- Abb. 5 47-jährige Frau mit - aus medizinischer Sicht - gesunden Beinen. Quelle: Dr. Tobias Bertsch, Földiklinik Hinterzarten.

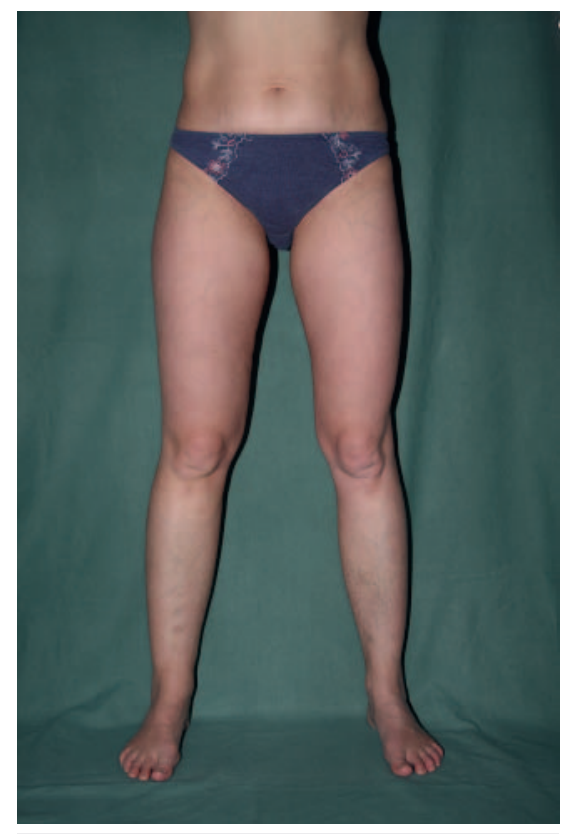

- Abb. 6 22-jährige Frau mit - aus medizinischer Sicht - gesunden Beinen. Quelle: Dr. Tobias Bertsch, Földiklinik Hinterzarten. 
nen, Ärztinnen, Wissenschaftlerinnen. Fünf Hände gingen in die Höhe, das entsprach in etwa auch der Gegenprobe. Dies macht eines deutlich: Wir haben es beim Thema „Frauenbeine“ sicher nicht regelmäßig mit einem medizinischen, sondern häufig auch mit einem gesellschaftlichen Phänomen zu tun.

Wir stimmen den Schreiberinnen des Leserbriefes zu, dass Frauen mit Lipödem Expertinnen ihrer eigenen Lebens- und Leidenswirklichkeit sind - genauso übrigens wie Frauen mit Depressionen, Brustkrebs, Lymphödemen oder Polyarthrose (ohne, dass wir uns hiermit auf eine „Was ist die schlimmere Krankheit“- Diskussion einlassen wollen). Allerdings - und das unterscheidet die „Lipödem-PatientinnenBewegung" von den genannten und allen anderen Erkrankungen - entscheiden dort Ärzte und wissenschaftlich fundierte Fakten über Krankheitskonzepte und Therapie und nicht Sichtweisen oder Wünsche von Patientinnen; Patientinnen, die (sicher auch aufgrund ärztlicher Fehlinformationen!) glauben, dass manuelle Lymphdrainagen und Liposuktion für das Lipödem zwingend notwendige Therapieoptionen darstellen.

\section{Zu 3.}

Die Leserbriefautoren schreiben: „Third, contrary to the view of this series, evidence of an edema component in lipedema continues to mount. Several imaging studies have shown damaged lymphatics in a significant proportion of women with lipedema independent of severity of their condition."

Hervorgehoben werden hier die Studie von Crescenzi et al. [26] sowie der gerade von Stanley Rockson et al. propagierte Biomarker PF4 [27].

In der Studie von Crescenzi wurden 10 Frauen mit der Diagnose Lipödem mittels spezialisierten MRT-Messungen untersucht. Eingeschlossen wurden hierbei auch Frauen mit einem sog. „Lipolymphödem" (eine Diagnose, die ohnehin nicht korrekt ist $[6,17,28]$, die aber verdeutlicht, dass die untersuchten Frauen additiv zum Lipödem auch an einem Lymphödem litten). Darüber hinaus wurden schwer adipöse Patientinnen mit einem maximalen BMI von $48,9 \mathrm{~kg} / \mathrm{m}^{2}$ eingeschlossen (in der Kontrollgruppe lag dieser nur bei 38,6 !), die per se schon aufgrund ihrer Adipositas ein deutlich erhöhtes Risiko für eine Lymphabflussschwäche haben [28]. Die Autoren der Studien fanden erhöhtes Sodium im Gewebe der untersuchten Patientinnen und stellten fest: „Skin sodium accumulation is an ermerging hallmark of inflammatory diseases and cardiovascular risk factor“ [26]. Dieser Befund stützt unsere in mehreren Publikationen dargelegte Auffassung, dass dem Lipödem eine „low grade inflammation “ des Fettgewebes zugrunde liegt [7, 10, 17].

Von den Ergebnissen dieser Untersuchung aber darauf zu schließen, dass das Lipödem eine lymphatische Dysfunktion und daraus folgend ein Ödem inkludiert; ein Ödem, welches mit MLD entstaut werden muss, ist ein gewagter pathophysiologischer Kunstgriff, der durch nichts belegt ist.

Auch der in Lipödem-Foren gerade propagierte PF4-Biomarker wurde im ersten US-amerikanischen Leserbrief bereits erwähnt. Damit soll, so die Autoren um Stanley Rockson von der Stanford University, gezeigt werden, „that in lipedema, lymphatic disfunction plays a role“ [27].

In einem Webinar, das von Lipedema Simplified (bzw. vom Lipedema Project) organisiert wurde, interviewt Catherine Seo, eine der Leserbriefverfasserinnen, Stanley Rockson zu diesem Thema.

Rockson zeigt hier eine von ihm als Lipödem diagnostizierte Patientin, die erhöhte PF4-Werte aufweist. Tatsächlich leidet die von ihm dargestellte Patientin vorrangig an einem ausgeprägten LYMPHÖDEM - vermutlich Adipositas-assoziiert, mit deutlicher Stauungsdermatose. Wir glauben gerne, dass bei DIESER Patientengruppe eine lymphatische Dysfunktion vorliegt. Die richtige Diagnosestellung, konkret die Differenzierung der Krankheit Lymphödem von der Krankheit Lipödem, ist aber unabdingbare Voraussetzung, um zu validen und seriösen Studienergebnissen zu kommen. Interessierte an diesem kurzen Videoclip von Rockson dürfen gerne den Erstautor dieser Replik um Zusendung bitten. Alternativ kann auch das gesamte Webinar von Catherine Seo angeschaut werden [29]

Darüber hinaus zeigen auch die anderen von den Leserbriefautoren in diesem Kontext aufgeführten Studien die bereits in unseren anderen Arbeiten genannten Schwächen [7, 17] und sind daher in keiner Weise geeignet, die gemachten Behauptungen zu untermauern.
So präsentieren in der Arbeit von Lohrmann et al. 8 der 13 untersuchten Patientinnen die nicht korrekte Diagnose „Lipolymphödem“, die aber darauf hinweist, dass auch Patientinnen mit Lymphödem und damit schon klinisch nachweisbarer Lymphabflussschwäche - in die Studie aufgenommen wurden [30]. Darüber hinaus wurden keine Angaben zum BMI der Patientinnen gemacht, sodass der Einfluss der Adipositas auf das Lymphödem unklar bleibt. In der zweiten aufgeführten Arbeit (Forner-Cordero I. et al) bekennen die Autoren selbst, dass „because of a lack of a test of diagnostic certainty of lipedema we may have included patients with lymphedema instead of lipedema and would justify the lymphoscintigraphic findings“ [31]. Die bei Forner-Cordero untersuchten Frauen waren bis 80 Jahre alt. Es ist gut belegt, dass mit zunehmendem Alter auch bei gesunden Menschen die lymphatische Funktion nachlässt. Entscheidend hierbei sind der altersbedingte Verlust der Glykokalyx sowie die vermehrte Produktion von proinflammatorischen Zytokinen, die die Permeabilität der Lymphgefäße erhöhen [32]. Auch bei der dritten von den Leserbriefverfassern angegebenen Arbeit [33] beträgt das Durchschnittsalter der Frauen 54,8 Jahre, der durchschnittliche BMI liegt bei 35,9, auch hier werden wieder Patientinnen mit der nicht korrekten Diagnose „Lipolymphödem“ untersucht - alles Kriterien, die die Aussagekraft erheblich einschränken. Überhaupt liest sich die Arbeit der Kollegen der Plastischen Chirurgie des Keck Medical Centers aus Los Angeles, die ihren Patientinnen mit Lipödem gerne gleich 2 Operationen anbieten, wie eine Werbeschrift: „A combination of lymphovenous anastomosis and debulking surgery, for expample, has been performed at our institution can offer improvement in physiologic drainage of excess lymphatic fluid and removal of excess adipose tissue, respectively“ [33]. Wie bereits an anderer Stelle vermerkt, gilt auch hier: "Money makes the world go round“ [1].

Noch einmal zum US-amerikanischen Lipödem-Weltbild und der angeblichen „evidence of an edema component in lipedema“.

Ödeme sind bekanntlich definiert als „abnorme Flüssigkeitsansammlung“ im Gewebe [34]. Bisher konnten allerdings weder klinische Untersuchungen noch Bildgebungen jemals ein relevantes Ödem 
bei Patientinnen mit Lipödem-Syndrom nachweisen [7]. Eine multizentrische Studie mit hochauflösendem Ultraschall bei Patientinnen mit der Diagnose Lipödem fand keinen Nachweis für Flüssigkeit im Weichteilgewebe der Beine [35]. In einer 2020 erschienenen Studie, in der Patientinnen mit Lipödem-Syndrom mittels MR-Lymphografie untersucht wurden, konkludieren die Autoren: „The fat tissue was homogenous, without any signs of edema in pure lipedema patients“ [36]. Auch in histologischen Untersuchungen wurde niemals die Präsenz eines Ödems beschrieben [7]. Reich-Schupke, Altmeyer und Stücker schrieben bereits 2012 in einem bemerkenswerten Artikel: „Der Begriff „Lipödem“ ist eigentlich irreführend, da es sich nicht um ein Ödem, also um eine Flüssigkeitseinlagerung im Gewebe handelt“ [37]. Dies wurde auch von den Autoren der niederländischen LipödemLeitlinie bestätigt, in der sie den Terminus Lipödem als unglücklich („unfortunate term“) beschreiben, da er Flüssigkeit im Gewebe suggeriert, wo keine Flüssigkeit zu finden ist [38]. Das European Lipoedema Forum - eine hochrangig besetzte internationale Expertengruppe aus 7 europäischen Ländern - resümierte in einem vielbeachteten Consensuspapier: „There is no scientific evidence that Lipoedema is an „oedema problem“ “ (Hervorhebung im Original) [10]. Dieser European Consensus wird inzwischen von Key Opinion Leaders aus 10 europäischen Ländern unterstützt [17]. Last but not least: Prof. Hugo Partsch, einer der renommiertesten Phlebologen weltweit, stellt in seinem Vorwort in dem inzwischen auch international publizierten International Consensus Document fest: „...lipoedema neither include oedema nor is there any evidence for lymphatic insufficiency. For this reason, decongestive lymphatic therapy is an inadequate treatment for patients with pure lipoedema“ [17].

\section{Zu 4.}

Einigkeit besteht erfreulicher darin, dass temporär durchgeführte Diäten unbedingt vermieden werden sollen. „Indeed, dietary intervention must constitute a lifelong change." Dieser Sichtweise stimmen wir grundsätzlich zu, allerdings zeigt die gelebte Wirklichkeit, dass diese lebenslange Umstellung auf gesunde Ernährung (und vor allem auch Ernährungsverhalten) nur den allerwenigsten Menschen mit der Krankheit Adipositas gelingt. Die Studienlage zur konservativen Therapie der Adipositas ist, man kann es nicht anders bezeichnen, desaströs.

So nehmen je nach Studie zwischen 80 und $99 \%$ aller Patienten, die auf konservative Weise Gewicht abnehmen, dieses Gewicht im Langzeitverlauf wieder zu [39-46]. Gerade bei Frauen führen Gewichtsreduktionsversuche, die im Jugendalter begonnen wurden, zu einer oft jahrzehntelang andauernden Diätspirale mit stetigem Gewichtsanstieg [47].

Beim Lipödem ist der Rat zur konservativen Gewichtsreduktion besonders tückisch. Studien der Universität Hohenheim haben ergeben, dass Frauen (im Gegensatz zu Männern) nach einer Gewichtsabnahme überproportional im Bereich der unteren Körperhälfte ihr Gewicht wieder zunehmen [48] - und wie oben dargelegt, nimmt die weitaus große Mehrheit der Diätteilnehmer ihr abgenommenes Gewicht wieder zu. Mit anderen Worten: Jede Empfehlung an die Lipödem-Patientin, Gewicht abzunehmen, erhöht das Risiko einer weiteren Fettgewebszunahme im Bereich der Beine - und damit auch das Risiko einer Zunahme der Beschwerden.

Dennoch empfehlen die Schreiber des Leserbriefes „metabolische Therapien (gemeint ist hier die Keto-Diät), „even for patients with lipoedema and a BMI of $>40 \mathrm{~kg} / \mathrm{m}^{2}$." Studien, die diesen Ansatz im Langzeitverlauf unterstützen, werden nicht genannt.

Stattdessen wird, wie bereits im Leserbrief von Karen Herbst and friends, die bariatrische Operation äußerst kritisch beurteilt.

„We disagree that, 'It has been shown that bariatric surgery is the most effective treatment for losing weight'. "Begründet wird diese ablehnende Haltung zur Adipositas-Chirurgie mit: „This is not our experience. "Es fällt auf, dass die Leserbriefschreiberinnen in diesem Abschnitt auffällig viele Formulierungen wie „in our experience“, „we believe“, „patients often say“ benutzen. Dafür, dass zu Beginn des Leserbriefes wissenschaftliches Vorgehen angemahnt wurde, erscheinen uns diese Glaubensbekenntnisse der Verfasserinnen bemerkenswert.
Darüber hinaus ist die Datenlage zum Langzeiterfolg der bariatrischen Chirurgie bei morbider Adipositas sehr konsistent und überzeugend [49-57]. Eine aktuelle Untersuchung des Universitätsklinikums Freiburg gemeinsam mit dem Europäischen Zentrum für Lymphologie in Hinterzarten zum Effekt der bariatrischen Operation bei schwer adipösen Patientinnen mit Lipödem-Syndrom zeigt den guten Erfolg dieser Therapieoption und bestätigt UNSERE seit 2008 bestehenden äußerst positiven Erfahrungen mit diesem Patientengut auch wissenschaftlich [58].

Die jetzt hier debattierten Differenzen dürfen nicht darüber hinwegtäuschen, dass es durchaus auch Gemeinsamkeiten in den Auffassungen der US-amerikanischen Lipödem-Akteurinnen und den unseren gibt.

Beide Autorengruppen sind sich einig darüber, dass die im Europäischen Consensus beschriebenen inflammatorischen Prozesse die pathophysiologische Grundlage des Lipödems darstellen, und dass psychische Belastungen sich negativ auf diesen inflammatorischen Verlauf auswirken können. Beide Autorengruppen sind darüber hinaus der Auffassung, dass ein Lipödem nichts mit Schuld oder Versagen der Patientin zu tun hat, wenngleich wir dies offener und direkter ansprechen. Dies erlaubt, die komplexe Beschwerdeproblematik (z. B. auch die Adipositas oder bestehende psychische Störungen) zielbewusst zu fokussieren.

Frauen mit Lipödem-Syndrom sind Expertinnen ihrer eigenen Lebens- und Leidenswirklichkeit - auch dem stimmen wir zu. Unser Bestreben liegt allerdings auch darin, Patientinnen - in dem bestehenden Chaos der Miss- und Desinformation zu diesem Krankheitsbild - nicht allein zu lassen, sondern sie auf dem Boden wissenschaftsbasierter Erkenntnisse in dieser Lebens- und Leidenswirklichkeit unterstützend zu begleiten.

Interessenkonflikt

Die Autorinnen/Autoren geben an, dass kein Interessenkonflikt besteht. 
Autorinnen/Autoren

\section{Tobias Bertsch, Gabriele Erbacher}

Europäisches Zentrum für Lymphologie im Schwarzwald, Földiklinik

\section{Korrespondenzadresse}

\section{Dr. Tobias Bertsch}

Földiklinik Hinterzarten - Europäisches Zentrum für Lymphologie tobias.bertsch@foeldiklinik.de

\section{Literatur}

[1] Bertsch T, Erbacher G. Response to the Letter to the Editor regarding Lipoedema - myths and facts, Part 1 and Part 5. European Best Practice of Lipoedema - Summary of the European Lipoedema Forum consensus. Phlebologie 2020; 49: 31-49

[2] Abrufbar unter: https://lipedemasimplified.org

[3] Abrufbar unter: https://lipedemaproject.org

[4] Abrufbar unter: https://lipedema-simplified. org/masterclass2020/

[5] Abrufbar unter: https://lymfsverige.se/ omoss.html

[6] Bertsch T, Erbacher G. Lipedema - myths and facts. Part 1. Phlebologie 2018; 47: 84-92

[7] Bertsch T, Erbacher G. Lipedema - myths and facts. Part 2. Phlebologie 2018; 47: 120 126

[8] Bertsch T, Erbacher G. Lipedema - myths and facts. Part 3. Phlebologie 2018; 47: 188 197

[9] Bertsch T, Torio-Padron N, Erbacher G. Lipedema - myths and facts. Part 4. Phlebologie 2019; 48: 47-56

[10] Bertsch T, Erbacher G, Corda D et al. Lipoedema: myths and facts, part 5. European best practice of lipoedema - summary of the European Lipoedema Forum consensus. 2020 https://doi.org/10.1055/a-1012-7670

[11] Abrufbar unter: http://www.lymfologi.se/ lipodem/

[12] Bertsch T, Martin KP. Obesity prevalence among lipoedema patients in a lymphological outpatient clinic with statutory health insurance in 2015 (unpublished data).

[13] Bosman J. Lipoedema: poor knowledge, neglect or disinterest? ] Lym- phoedema 2011; 6 (2): 109-111

[14] Child AH, Gordon KD, Sharpe P. Lipedema: an inherited condition. Am J Med Genet A 2010; 152A (4): 970-976. https://doi.org/ 10.1002/ ajmg.a.33313

[15] Herbst KL, Mirkovskaya L, Bharhagava A et al. Lipedema fat and signs and symptoms of illness, increase with advancing stage. Arch Med 2015; 7: 10

[16] Dudeck JE, Białaszek W, Ostaszewski P et al. Depression and appearance related distress in functioning with lipedema. Psychol Health
Med 2018; 23 (7): 846-853. https://doi.org/ 10.1080/13548506.2018.1459750

[17] Bertsch T, Erbacher G, Elwell R et al. Lipoedema: a paradigm shift and consensus. J Wound Care 2020; 29 (Suppl. 2): S1-S52

[18] Erbacher G, Bertsch T. Lipoedema and pain: what is the role of the psyche? Results of a pilot study with 150 lipoedema patients. Phlebologie 2020; 49 (5): 305-316. https://doi.org/10.1055/a-1238-6657

[19] Linsmayer D, Neidlinger PK, Braus DF. Rheuma und Psyche - Eine Kurzübersicht. Orthopäde 2019; 48 (11): 957-962. doi:10.1007/s00132-019-03812-8

[20] Baerwald C, Manger B, Hueber A. Depression als Komorbidität bei rheumatoider Arthritis. Z Rheumatol 2019; 78: 243-248. https://doi.org/10.1007/s00393-018-0568-5

[21] Hofmann P, Hemberger S, Lunzer R et al. Psychische Aspekte der rheumatoiden Arthritis Wie ansprechen? Wie behandeln? Zusammenfassung des Expertenmeetings „Rheuma trifft Psyche“ am 05.11.2015. Abrufbar unter: ttps://www.pfizermed.at/ sites/default/files/rheuma_trifft_psyche.pd

[22] Bischoff N, Morina N, Egloff N. Chronischer Schmerz bei Traumatisierung. Komplexität und Herausforderung bei Diagnostik und Therapie. PiD - Psychotherapie im Dialog 2016; 17 (4): 69-72. doi:10.1055/ s-0042-116706

[23] Viana MC et al. Prior mental disorders and subsequent onset of chronic back or neck pain: findings from 19 countries. J Pain 2018; 19 (1): 99-110. doi:10.1016/j.jpain.2017.08.011

[24] Tegethoff M, Belardi A, Stalujanis E. Comorbidity of Mental Disorders and Chronic Pain: Chronology of Onset in Adolescents of a National Representative Cohort. The Journal of Pain 2015; 16 (10): 1054-1064. doi:https://doi.org/10.1016/j.jpain. 2015.06.009

[25] Hooten WM. Chronic Pain and Mental Health Disorders. 2016 Mayo Foundation for Medical Education and Research, Mayo Clin Proc 2016; 91 (7): 955-970

[26] Abrufbar unter: https://www.mayoclinic proceedings.org/article/S0025-6196(16) 30182-3/pdf

[27] Crescenzi R, Marton A, Donahue PMC et al. Tissue Sodium Content is Elevated in the Skin and Subcutaneous Adipose Tissue in Women with Lipedema. Obesity 2017; 26: 310-317. doi:org/10.1002/oby.22090

[28] Ma W, Gil HJ, Escobedo N et al. Platelet factor 4 is a biomarker for lymphatic-promoted disorders. JCI Insight 2020; 5: 13e135109. doi:10.1172/jci.insight.135109

[29] Bertsch T. Obesity-related lymphoedema underestimated and undertreated. Phlebologie 2018; 47 (2): 75-83

[30] Lipedema simplified, webinar: Abrufbar unter. https://lipedema-simplified.org/ webinar2020-pf4/; hier Minute 14:37-14:47

[31] Lohrmann C, Foeldi E, Langer M. MR imaging of the lymphatic system in patients with lipedema and lipo-lymphedema. Microvasc
Res 2009; 77: 335-339. doi:org/10.1016/ j.mvr.2009.01.005

[32] Forner-Cordero I, Oliván-Sasot P, RuizLlorca $C$ et al. Hallazgos linfogammagráficos en pacientes con lipedema. Rev Esp Med Nucl Imagen Mol 2018; 37: 341-348. doi:org/10.1016/j.remnie.2018.10.013

[33] Shang T, Liang J, Kapron C et al. Pathophysiology of aged lymphatic vessels. Aging 2019; 11 (16): 6602-6613

[34] Gould DJ, El-Sabawi B, Goel P et al. Uncovering Lymphatic Transport Abnormalities in Patients with Primary Lipedema. J Reconstr Microsurg 2019; 36: 136-141. doi:10.1055/ s-0039-1697904

[35] Abrufbar unter: https://www.pschyrembel. de/Ödem/KOFKR

[36] Hirsch T, Schleinitz J, Faerber G et al. Is the differential diagnosis of lipoedema by means of high-resolution ultrasonography possible? Phlebologie 2018; 47 (4): 182-187

[37] Cellina M, Gibelli D, Soresina M et al. Noncontrast MR Lymphography of lipedema. Magent Resonance Imaging 2020; 71: 115124

[38] Reich-Schupke S, Altmeyer P, Stücker M. Thick legs - not always lipedema. J Dtsch Dermatol Ges 2013; 11 (3): 225-233

[39] Lipedema - Guidelines in the Netherlands 2014. Abrufbar unter https://www.gdlymph. eu/assets/pdf/Dutch-lipoedema-guideline2014.pdf

[40] Stunkard A. The Results of Treatment for Obesity. A Review of the Literature and Report of a Series. AMA Arch Intern Med 1959; 103 (1): 79-85

[41] Bennett W, Gurin J. The Dieter's Dilemma: Why Diets Are Obsolete-the New Setpoint Theory of Weight Control. New York: Basic Books; 1982

[42] Cogan J, Rothblum E. Outcomes of weightloss Programs. Genetic, Social and General Psychology Monographs 1993; 118: 4

[43] Perri MG, Nezu AM, Patti ET et al. Success and failure in the treatment of obesity: Where do we go from here? Medicine, Exercise, Nutrition and Health 1995; 4: 255-272

[44] Hensrud DD, Weinsier RL. A prospective study of weight maintenance in obese subjects reduced to normal body weight without weight-loss training. American clinical Nutrtion 1994; 60 (5): 688-694

[45] Mann T, Tomiyama AJ, Westling E. Medicare's search for effective obesity treatments: diets are not the answer. Am Psychol 2007; 62 (3): 220-233

[46] Nordmann A, Bucher H. Effects of low carb vs low fat diets on weight loss and cardiovascular risk factors: a meta-analysis of randomized controlled trials. Arch Internal Med 2006; 166 (8): 285-293

[47] Fildes A, Charlton J. Probability of an obese person attaining normal body weight. American Journal of Public Health 2015; 105 (9): e54-e59 
[48] Pietiläinen KH, Saarni SE, Kaprio ]. Does Dieting Make You Fat? International J of Obesity 2012; 36 (3): 456-464

[49] Bosy-Westhpal A. Effect of weight loss and regain on adipose tissue distribution, composition of lean mass and resting energy expenditure in young overweight and obese adults. Int J Obes (Lond) 2013; 37 (10): 1371-1377

[50] Buchwald H, Estok R, Fahrbach K et al. Weight and type 2 diabetes after bariatric surgery: systematic review and metaanalysis. Am J Med 2009; 122: 248-256

[51] Wittgrove AC, Clark GW. Laparoscopic Gastric Bypass, Roux-en-Y - 500 Patients: Technique and Results, with 3 - 60 months follow-up. Obes Surg 2000; 10: 233

[52] Sugerman HJ, Londrey GL, Kellum JM et al. Diabetes and hypertension in severe obesity and effects of gastric bypass-induced weight loss. Ann Surg 2003; 237: 751-756

[53] Rasheid S, Banasiak M, Lipska A et al. Bypass is an Effective Treatment for Obstructive Sleep Apnea in Patients with Clinically Significant Obesity. Obes Surg 2003; 13: 5
[54] Courcoulas AP, Yanovski S, Arterburn DE et al. Long-term Outcomes of Bariatric Surgery: A National Institutes of Health Symposium. JAMA Surg 2014; 149 (12): 1323-1329

[55] Arterburn DE, Olsen M, Maciejewski M et al. Association Between Bariatric Surgery and Long-term Survival. JAMA 2015; 313 (1) 62-70

[56] Sjöström L, Nabro K, Sjöström D et al. Effects of bariatric surgery on mortality in Swedish obese subjects. N Engl J Med 2007; 357: 741-752

[57] Adams TD, Gress R, Hunt S et al. Long-term mortality after gastric bypass surgery. N Engl J Med 2007; 357: 753-761

[58] Shubeck S, Dimmick J, Telem D. Long-term Outcomes Following Bariatric Surgery. JAMA 2018; 319 (3): 302-303

[59] Fink JM, Schreiner L, Bertsch T. Leg volume in patients with lipedema following bariatric surgery. Visc Med 2020. doi:https://doi.org/ $10.1159 / 000511044$
Publikationshinweis

Leserbriefe stellen nicht unbedingt die Meinung von Herausgebern oder Verlag dar. Herausgeber und Verlag behalten sich vor, Leserbriefe nicht, gekürzt oder in Auszügen zu veröffentlichen.

\section{Bibliografie}

Phlebologie 2021; 50: 14-20

DOI 10.1055/a-1302-9212

ISSN 0939-978X

(C) 2021. Thieme. All rights reserved. Georg Thieme Verlag KG, Rüdigerstraße 14, 70469 Stuttgart, Germany 